\title{
Review of the Effects of Stationary/Rotating Cylinder in a Cavity on the Convection Heat Transfer in Porous Media with/without Nanofluid
}

\author{
Ahmed Dhafer Abdulsahib, Khaled Al-Farhany* \\ Department of Mechanical Engineering, University of Al-Qadisiyah, Al-Qadisiyah 58001, Iraq
}

Corresponding Author Email: Khaled.alfarhany@qu.edu.iq

https://doi.org/10.18280/mmep.080304

Received: 19 May 2020

Accepted: 12 April 2021

\section{Keywords:}

mixed convection, nanofluid, porous medium, two layers, circular cylinder, rotating cylinder

\begin{abstract}
The mixed convective heat transfer has many multiple engineering applications, such as solar collectors, electronic equipment cooling and heat exchanger, and geothermal engineering. This work presents comprehensive coverage of a wide range of published studies in terms of convection heat transfer inside the enclosure in recent years. The convective heat transfer in porous media with/without nanofluid, and the effect of stationary/rotating cylinder inside cavities, as well as the position of the cylinder, had been addressed and discuss to draw the main conclusions and recommendations.

It is worthy to mention that the mixed convective with the effect of entropy generation with inner bodies in enclosure has been investigated less than the other simple enclosure shapes due to its complexity. The researchers can be extended their future studies by adding the MHD effects with mixed conviction in simple/complex shapes of enclosure. This study gives an important and useful summary for provides researchers of heat transfer in academic and industrial. At the end of this investigation, the governing equations of the 2-D mixed convection in an enclosure filled with porous medium, and nanofluid addressed.
\end{abstract}

\section{INTRODUCTION}

The studies of enhancement convection heat transfer inside the cavities have appeared the attention of researchers and study what are the parameters affected on heat transfer because of its multiple engineering applications, for example, nuclear reactor, solar collector, electronic equipment cooling, and heat exchanger [1-5]. Figure 1 shows some of these applications. Enhanced by adding solid nanoparticle to base fluid, developing the enclosure by repair its geometry, adding fins or baffles and inserting objects inside the enclosure in the fixed case or rotating case, etc. Also, the interest in multi-layer flow has appeared in the sixties of the twentieth century due to the wide applications in the industry. However, the flow passing through the porous media with the associated heat transfer has been interested in the scientists and engineers for centuries, because involved in a lot of recent industrial and environmental applications [6] shows some of these applications.

In this study, discuss the literature review of researchers. It was divided into five sections according to the working fluid and used porous material and according to the insertion of objects inside the cavity in a fixed or rotating state.

\section{HEAT TRANSFER INSIDE CAVITY WITH NANOFLUID}

Santra et al. [7] explained the effect of the use of a nanofluid (water/copper) as a cooling working fluid on the laminar convection heat transfer in a square cavity. The right and left vertical walls were differentially heated and the top and bottom walls were insulated. The finite volume method was implemented to solve the mathematical equations of nonNewtonian fluids by using the SIMPLER algorithm.
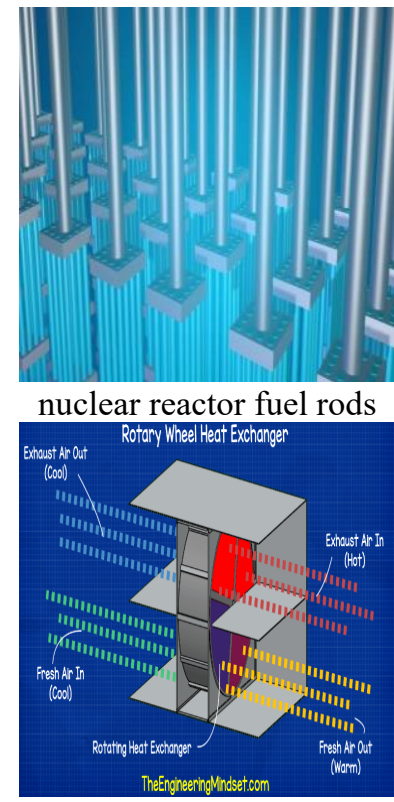

HVAC heat exchangers

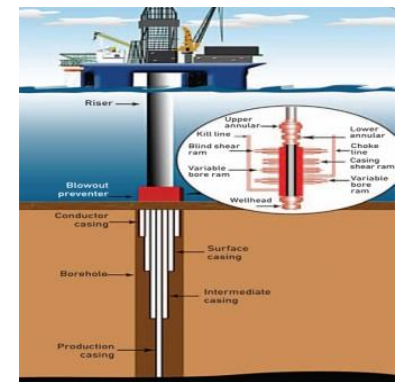

drilling of oil wells

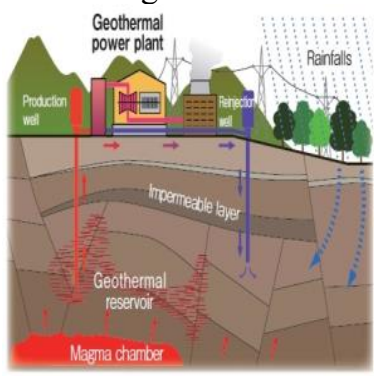

geothermal reservoirs
Figure 1. Present study applications

The study used the following parameters; Rayleigh number (Ra) with ranged from $10^{4}$ to $10^{7}$, the concentration of the copper particles in water $(\phi)$ ranged from $0.05 \%-5 \%$, with nanoparticles diameter around $100 \mathrm{~nm}$. The results showed that the heat transfer rate increases by increasing Ra and $\phi$. Ho et al. [8] investigated the effects of the thermal conductivity 
and dynamic viscosity of a nanofluid on the natural convection inside a square cavity. Numerical simulations were done for a homogeneous solid-liquid mixture of alumina and water. The bottom and the top walls are assumed to be adiabatic, and the other walls were heated differently. Two main parameters were considered namely Rayleigh number $\left(10^{3} \leq \mathrm{Ra} \leq 10^{6}\right)$ and the volumetric fraction of solid particles $(0 \leq \phi \leq 0.04)$. The results showed that the dynamic viscosity and effective thermal conductivity of the considered nanofluid have a strong influence on the properties of the atural convection inside the cavity. Abu-Nada and Oztop [9] studied numerically the effects of the inclination angle on fluid flow and heat transfer in a cavity filled with nanofluid. The nanofluid performance inside the cavity is tested through account dispersion of solid particles. The transferred heat and the flow characteristics vary by the variations of the inclination angle ranged from $0^{\circ}$ to $120^{\circ}$. Finite-volume methods used to solve the governing equations with Rayleigh number $\left(10^{3} \leq \mathrm{Ra} \leq 10^{5}\right)$. It was found that the effect of concentration of nanoparticle on Nusselt number was more obvious at low volume fraction compared with the cases of high volume fraction. The enhancement percentage of heat transfer is decreased when using nanoparticles at higher $\mathrm{Ra}$. The fluid flow and the heat transfer of natural convection in a square enclosure filled with $\mathrm{Al}_{2} \mathrm{O}_{3}$ water were studied numerically by Lin and Violi [10]. The right and left walls were differentially heated and the upper and lower walls were insulated. The rates of heat transfer were investigated for different parameters which are irregular nanoparticle size, volume fraction, Grachoff number, the average nanoparticles diameter, and Prandtl number. The results showed that the decrement in Prandtl number leads to expand the effects of nanoparticles because of the increased effective thermal diffusion. Ternik and Rudolf [11] studied numerically the promotion of heat transfer due to the addition of various nanoparticles $\left(\mathrm{TiO}_{2}, \mathrm{Al}_{2} \mathrm{O}_{3}, \mathrm{Au}\right.$, and $\left.\mathrm{Cu}\right)$ in water. This study was done in a two-dimensional cavity with Rayleigh number $\left(10^{3} \leq \mathrm{Ra} \leq 10^{5}\right)$ and the concentration of nanoparticles $(0 \leq \phi \leq 0.010)$. The finite volume method was adopted to solve the governing equations. It was found that the average Nusselt number increases with increasing the Rayleigh number and the volume fraction. The authors exhibited that the improvement of heat transfer can be achieved using the considered nanofluids compared to the classic fluid which showing more enhancement in low Rayleigh numbers than high Rayleigh numbers. Jahanshahi and Hosseinizadeh [12] studied numerically enhancing the heat transfer in a square enclosure by using ( $\mathrm{SiO} 2$-water) nanofluid. The temperature of the vertical walls are different, while other walls consider adiabatic. The nanofluid conductivity value was extracted experimentally. The Rayleigh number $\left(10^{3} \leq \mathrm{Ra} \leq 10^{7}\right)$ and the volumetric fraction of nanoparticles $(0 \leq \phi \leq 0.04)$ parameters were used in their study. The findings showed that the average Nusselt number increases when increasing the volume fraction for all values of the Rayleigh number. Oztop, Mobedi et al. [13] investigated the path of heat transfer in an inclined cavity having a square shape and filled with nanofluid (CuO-water). One wall of the cavity was hot and the opposite wall was cold while the remaining walls were insulated. Continuity, momentum, and energy equations were solved by using the finite volume method. The study was conducted at $\left(10^{3} \leq \mathrm{Ra} \leq 10^{5}\right)$, inclination angle $(0,30,60$ and 90$)$ and the concentration of nanoparticle $(0,0.02,0.04,0.06,0.08$ and 0.1$)$. The results indicated that the transferred heat inside the enclosure increased by using nanoparticles which reaching the highest rates at lower Rayleigh number values. Barik and Al-Farhany [14] studied the effects of baffle angle on natural convective heat transfer inside a square cavity filled with nanofluid. The results show that the highest Nusselt number occurs when the baffle angle equal to $90^{\circ}$. Chopkar et al. [15] showed a significant increase of up to 100 pct with only $1.5 \mathrm{vol}$ pct nanoparticles of 30- to 40-nm average diameter.

\section{HEAT TRANSFER INSIDE CAVITY WITH POROUS MEDIA}

A numerical analysis of the convection heat transfer in a cavity having a square shape and contains a porous medium was implemented by Sathiyamoorthy et al. [16]. The lower wall was heated while the upper wall was isolated. Other side walls were taken in two cases; the first case both walls were heating, and the second case includes a linear heating of the left wall and keeping the right wall at cold temperature. The parameters used in their study were Rayleigh number $\left(10^{3} \leq \mathrm{Ra}\right.$ $\left.\leq 10^{6}\right)$, Prandtl number $(0.2 \leq \operatorname{Pr} \leq 100)$, and Darcy number $\left(10^{-}\right.$ $\left.{ }^{5} \leq \mathrm{Da} \leq 10^{-3}\right)$. The results showed that the increase of Rayleigh and Darcy numbers cause increasing the average Nusselt number. Oztop [17] studied numerically normal convection in a tilted rectangular enclosure filled with porous media. One wall was partially cooled and the other one was fully heated while the other walls were insulated. The finite element method was adopted for solving the governing equations. The author used the following parameters; Rayleigh number ( $10 \leq$ $\mathrm{Ra} \leq 1000)$, length of cooler $(0.25 \leq \mathrm{w} \leq 0.75)$, the heated location center $(0.1 \leq \mathrm{c} \leq 0.9)$, and the inclination angle $\left(0^{\circ} \leq\right.$ $\left.\Theta \leq 90^{\circ}\right)$. It was concluded that the heat transfer rate was enhanced with the increase in $\mathrm{Ra}$, while it reduced with increasing the aspect ratio. Revnic et al. [18] studied the normal convection for an inclined-porous enclosure. The right and bottom cavity walls were insulated, while the other left and top walls were heated and cooled, respectively. A central finite difference scheme was implemented for solving the governing equations. The studied parameters are the inclination angle of the cavity $(0 \leq \Theta \leq 315)$ and Rayleigh number $(10 \leq \mathrm{Ra} \leq 1000)$. The authors found that at $\Theta=-45^{\circ}$ and $\mathrm{Ra}=100$, the average Nusselt number is the lowest value. Al-Farhany and Turan [19] studied the natural convection in an inclined enclosure. Adiabatic conditions were assumed for the top and bottom walls. The right and the left walls were assumed to be cooled and heated, respectively. The considered parameters were; two values of Rayleigh number $\left(34 * 10^{4} \leq \mathrm{Ra}\right.$ $\left.\leq 3.8^{*} 10^{4}\right)$, aspect ratio ( 5 to 32.7$)$, the inclination angle $\left(0^{\circ} \leq\right.$ $\left.\Phi \leq 90^{\circ}\right)$, and modified Prandtl number $(3.1 \leq \operatorname{Pr} \leq 499)$. The mathematical equations were solved by the DarcyForchheimer-Brinkman model. It was concluded that the increment in the isotherms, streamline, and average Nusselt number can be obtained by increasing the Rayleigh number and the angle of inclination. A numerical analysis of the conjugate natural convection characteristics in a cavity contains a porous medium was presented by Abdulkadhim et al. [20]. The horizontal walls of the enclosure considered adiabatic while the right and left walls are heated and cooled respectively. The finite element method was adopted to solve the governing equations. The considered parameters were Rayleigh number $\left(10 \leq \mathrm{Ra} \leq 10^{3}\right)$, Darcy number $\left(10^{-3} \leq \mathrm{Da} \leq\right.$ $\left.10^{-1}\right)$, aspect ratio $(0.5 \leq \mathrm{AR} \leq 10)$, and Prandtl number $(\mathrm{Pr}=$ $0.71)$. The results showed that for all cases the stream function 
values increased by increasing Rayleigh number. Also, the locations of partially active walls affect the heat transfer rate. Al-Farhany and Abdulkadhim [21] numerically studied characteristics of conjugate convective heat transfer in a cavity containing a porous medium. The Top and bottom walls as well as the part of the left walls were insulated. Also, the part of the left wall was heated, and remaining the right wall was cooled. The equations of non-Newtonian fluids were numerically solved by COMSOL Multiphysics. The main parameters were Grashof number, Darcy number, modified Rayleigh number, and thermal conductivity ratio. The results showed that the increase of modified Rayleigh number causing in increasing the circulation strength, Furthermore, the increase in heat source length causing in increase the local Nusselt number. Also, Al-Farhany and Abdulkadhim [22] shows that when the wall thickness increases, the Nusselt number decreases while it increases when the modified Rayleigh number increases. The conjugate natural convection studied numerically by Abdulkadhim et al. [23]. It is found that when the Wall thickness increases, the conduction heat transfer dominated. The heat transfer rate reduced When increasing aspect ratio.

\section{HEAT TRANSFER INSIDE CAVITY WITH NANOFLUID AND POROUS MEDIA}

The natural convection heat transfer and fluid flow inside an enclosure separated vertically into a fluid area and a saturated porous area was studied by Tong and Subramanian [24]. The parameter range used in this study were; Rayleigh number between $10^{4}$ and $10^{5}$, Darcy number from $10^{-3}$ to $10^{-7}$, and the aspect ratio varies as 5, 10, and 20. The finite difference forms were used to solve the governing equations. The boundary between the porous and fluid is impermeable matching conditions of the shear stress that are obtained using Brinkman's extension model. It was noticed that under several considerations, there is no need to fill the enclosure with a porous medium to get the optimal effect of insulation. Tatsuo et al. [25] described an investigation of heat transfer by natural convection in a cavity divided into porous and fluid layers. The Brinkman extension of Darcy low is used to hold within the porous region. The range of this study was $\left(10^{3} \leq \mathrm{Ra} \leq 10^{5}\right)$ and $\left(10^{-3} \leq \mathrm{Da} \leq 10^{-5}\right)$. The results illustrated that the stream functions and isotherms clearly changed with Rayleigh number and Darcy number. Sathe et al. [26] assumed numerically that the interface between the porous and fluid regions is permeable. The power low finite difference method was utilized for solving the equations including the following parameters; $\left(\mathrm{Ra}=10^{3}\right.$ to $\left.10^{6}\right),\left(\mathrm{Da}=10^{-2}\right.$ to $\left.10^{-5}\right)$, and (Aspect ratio $=5,10$, and 20). The effect of the selected parameters (Da, $\mathrm{Ra}$, and $\mathrm{A}$ ) on the Nusselt number calculated and compared in two different cases; the first one when the interface wall was permeable and the second case when it was impermeable. It was found that the heat transfer inside the enclosure for the second case higher than the first case. Campos et al. [27] numerically investigated the heat transfer by free convection and fluid flow in an annular cavity that was partly filled with porous media. The heat transfer rate across the annular porous/fluid section was the lowest by filling it partially with a porous medium. The results revealed that the Nusselt number showed a different trend with the variation of the width of the porous media in the enclosure $(\mathrm{XP}=0.1,0.3,0.5,0.7$, and 0.9$)$. The best conditions of insulation are gained with porous partially filled with the material. Bagchi and Kulacki [28] investigated the free convection in a cavity filled with fluid and porous layers that were subjected to heat from the bottom side. The investigated parameters were; Rayleigh number $\left(10^{3}\right.$ to $10^{6}$ ), overall layer height (between 0 and 1 ), a heater to cavity length ratio $(0.25,0.5,1)$, porous layer to cavity height ratio $(0.25,0.5,0.75)$, domain aspect ratio $(2,4,6)$, and Darcy number $\left(10^{-2}, 10^{-4}, 10^{-6}\right)$. The result illustrated that the Nusselt number rise with a reduction in the height ratio and length of the heater, and rise with the Darcy number. Alsabery et al. [29] examined numerically the unsteady natural convection using COMSOL software. The finite element method solves the governing equations of porous and fluid layers. It was shown that the maximum value of stream function ( $\psi$ max) in the porous layer decreased by increasing the time, while it is increased in the fluid layer. Also, it was clarified that the distortion of the isotherms porous layer increased by increasing the time, and reached a steady-state, while the isotherms fluid layer becomes parallel to the vertical wall by increasing the time. Al-Jasim [30] explored numerically the natural free convection inside an enclosure having a square shape. The two layers of the nanofluid $\left(\mathrm{TiO}_{2}\right.$-water) and the porous medium were filled with the same nanofluid in the cavity. The governing equations solved by the finite element method. Applied partly uniform constant heat flux at the left vertical wall and the right vertical wall was kept at constant cold temperature, while other walls were considered adiabatic. The range of parameters were; Rayleigh number $\left(10^{4} \leq \mathrm{Ra} \leq 10^{7}\right)$, heat generation constant $(0 \leq \mathrm{k} \leq 20)$, Darcy number $\left(10^{-1} \leq \mathrm{Da} \leq 10^{-5}\right)$, and volume fraction $(0 \leq \varphi \leq 0.1)$. The results indicated that the maximum stream function ( $\psi$ max) decreased as the thickness of the porous layer increased. Laminar natural convection flow in a square enclosure having a vertical sinusoidal interface between the porous layer and the nanofluid layer was studied numerically by Hakim et al. [31]. The authors used the finite difference method available with COMSOL Multiphysics software. The Darcy-Brinkman model has been used to solve the governing equations in the non-uniform porous layer. The selected parameters in their study were the non-uniform porous layer thickness, Rayleigh number, Darcy number, sinusoidal amplitude, and the number of undulations. The results showed that the low value of the porous thickness significantly improves the rate of heat transfer inside the considered enclosure. It was concluded that increasing $\mathrm{Ra}$ and $\mathrm{Da}$ leads to a clear enhancement in the average Nusselt number. Al-Amir et al. [32] studied numerically the influence of Prandtl number in a square enclosure. This enclosure was filled by two layers of Ag-water as a porous medium and saturated with a non-Newtonian fluid and nanofluid separated by a vertical interface of sinusoidal shape. The wall at the left side was subjected to heat, while the wall located at the left side was cooled. The upper and lower walls were reflected adiabatic. Galerkin finite-element method has been used to solve the governing equations. A wide range of parameters have been used including; Darcy number within the range of $\left(10^{-5}<\mathrm{Da}<10^{-1}\right)$, power index $(1.4>\mathrm{n}>0.6)$, undulation number $(1<\mathrm{N}<4)$ and volume fraction $(0<\phi<0.2)$ on cavity thermal performance for Prandtl numbers $(0.015$ to 13.4), and Rayleigh number equal to $10^{5}$. The results presented that the average Nusselt number increases when Prandtl and Darcy numbers increasing, while it is reducing with the increasing power index (n). For the shear thinning behavior of the nanofluid at $\operatorname{Pr}$ equal to 13.4 and $\mathrm{n}$ equal to 0.7 , the influence of the number of undulations on average Nusselt number is not apparent. 


\section{STUDIES OF THE HEAT TRANSFER INSIDE CAVITY WITH A CIRCULAR CYLINDER}

Yoon et al. [33] studied numerically the free convection due to the difference in the temperature between an outside square enclosure (cold) and an internal circular cylinder (hot) at constant Rayleigh number $\left(\mathrm{Ra}=10^{7}\right)$. The influence of the location of the inner circular cylinder on the fluid flow and heat transfer was investigated. The results presented that the value of the Nusselt number increase with the change of upside and downside position of the inner cylinder. Hussain and Hussein [34] provided a numerical study of a steady state free convection for an inner cylinder subjected to a heating source applied inside a cold square cavity filled with air. The finite volume method was utilized to solve the governing equations. Rayleigh number and the position of the inner cylinder are the main parameters of their study. The result showed that the local and average Nusselt number values increase as the Rayleigh number increases at different positions of the inner cylinder. That's happened because of its big impact on thermal convection. Hussein [35] Ali investigated numerically the natural convection in an air-filled parallelogram enclosure with a heated circular concentric cylinder. The cavity's left and right sidewalls are cooled, while its bottom and top walls are considered thermally insulated. The finite volume numerical simulation was used for the simulation. The selected parameters were Rayleigh number $\left(10^{4}<\mathrm{Ra}<10^{7}\right)$, the cylinder vertical distance $(-0.2$ to 0.2$)$, and the angle of inclination ( 0 to 30$)$. The results indicated that the intensity of the circulation of the flow and the developed thickness of the thermal boundary layer close to the hot boundary of the circular cylinder was increased affectedly as the Rayleigh number increased. In order to increase the circulation of the flow inside the enclosure, the inner cylinder should be moved down exactly right at -0.2 when the sidewalls of the parallel ogrammic enclosure aligned within $15 \mathrm{o}$. Abdulkadhim et al. [36] study natural convection square porous enclosure with a circular cylinder. The results show that the heat transfer rate is dependent on the diameter and location of the cylinder. Ravnik and Skerget [37] implemented a numerical simulation of the flow and heat transfer of the heated circular/elliptical cylinders in the cooled cubic cavity using nanofluids. The properties of nanofluids were constant across the domain for all nanoparticles with volume fractions of 0.1 and 0.2 . The results display that the use of nanofluid leads to enhance the heat transfer, where convection was the dominant heat transfer mechanism. In addition, the heat transfer characteristics in the elliptical case is slightly better by comparing the circular and elliptical cylinders. Hatami and Safari [38] studied numerically the natural convection of nanofluid for heated cylinder inside a wavy-wall enclosure. The authors studied different locations of the cylinder along the cartesian coordinate of the model. It was concluded that the central location of the cylinder assists the heat transfer in both wavy sidewalls.

\section{HEAT TRANSFER INSIDE CAVITY WITH A ROTATING CIRCULAR CYLINDER}

Lewis [39] performed a numerical analysis of a steady, incompressible, viscous flow between the inner circular cylinders and bounded square cavity. The rotational motion of the circular cylinder represented the driver force of the flow inside the square cavity. The results were provided for the Reynolds number with the range of 1-1400 and different effective parameters, such as the length of the square side to the circle diameter ratio. Fu et al. [40] examined numerically the heat transfer enhancement by the rotational motion of a cylinder inside a square cavity. The upper and the lower were adiabatic, while, the left and right walls were considered heated differently. The FEM had been used to solve the governing differential equations and the Newton-Raphson method was used to find the convergence solution. The results showed that the convection heat transfer is significantly affected by the rotating cylinder when only the counterclockwise direction is considered. The heat transfer enhancement was about $60 \%$ at the value of $\mathrm{Gr} / \mathrm{Re}^{2}$ is $10^{3}$. Misirlioglu [41] performed a numerical analysis of the heat transfer by convection in a square enclosure filled with a porous medium saturated by a fluid. The heat transfer was changed when the rotating cylinder located in the center of the enclosure. The cylinder diameter to cavity height is 0.8 . The input data used in the study were Grashof number of 106, the $\mathrm{Gr} / \mathrm{Re}^{2}$ between $\left(0.0625\right.$ and $\left.10^{2}\right)$, and the Darcy number of $\left(10^{-2}, 10^{-3}\right.$, and $\left.10^{-4}\right)$. To solve the governing equations, Galerkin finite element method was used. The consequence showed that the rotational motion in the forced convection is more active than that corresponding in mixed and normal convection. For the high speeds, the heat transfer and the Darcy number are independent. Costa and Raimundo [42] studied numerically mixed heat convection in a square cavity in the presence of a rotating cylinder placed in the middle. The effects of force and free convection can be combined due to the rotation cylinder. The rotating cylinder was affected in thermal heat transfer without imposing a thermal condition on its surface. The effect of the rotating cylinder on the subsequent mixed convection case was investigated by its radius, thermal conductivity, rotational velocity, and thermal capacity. The isotherms and flow structure visualize the thermal region and streamlines respectively. The results included the effects of the rotating cylinder on the cavity thermal performance. As an overall thermal transfer process, the cylinder's physical thermal properties are very important. Hussain and Hussein [43] investigated numerically a laminar steady mixed convection in a two-dimensional square cavity with a rotating circular cylinder by using a finite volume scheme. The left and opposite side walls are heated and cooled, respectively. The lower and the upper cavity walls are insulated. The considered cavity filled with air at a Prandtl number of 0.71 . The stream function, isothermal lines, and the average Nusselt number are given for Richardson numbers $(0$, 1, 5, and 10) and Reynolds numbers (50, 100, 200, and 300). The study included the effects of different locations of a circular cylinder and the ratios of the thermal conductivity on heat transport. The results showed that when the Richardson number and Reynolds number increase the average Nusselt number increases. The maximum heat transfer is achieved at a high concentration of nanoparticles with a high conductivity value, as well as slow positive rotation and a small cylindrical size in the cavity center. Roslan et al. [44] studied numerically the convective heat transfer in a square cavity with an inner rotating cylinder. The left surface was heated and the right surface was cooled, while the other walls and rotating cylinder were kept adiabatic. The medium of the cavity was filled by water as a base fluid with different types of nanoparticles ( $\mathrm{Ag}$, $\mathrm{Cu}, \mathrm{Al}_{2} \mathrm{O}_{3}, \mathrm{TiO}_{2}$ ). The governing equations were solved by COMSOL software. Several parameters were considered in 
this study, such as the solid volume fraction, the angular rotational speed, and the cylinder radius. The results showed that the thermal conductivity can be enhanced by increasing the nanoparticles concentration. The flow circulation strength can be increased by using a smaller cylinder with a high rotating speed in the negative rotational direction. The maximum value of the heat transfer was achieved at a higher nanoparticle concentration with a good conductivity value and a slowly rotating cylinder in the positive rotation for a small cylindrical size located at the center of the cavity. Matin and Pop [45] examined numerically the mixed convection of $\mathrm{Al}_{2} \mathrm{O}_{3}$-water nanofluid as a working fluid flows inside an eccentric horizontal annulus with a rotating inner cylinder. The inner cylinder was heated while the outer cylinder was cooled. The finite volume method was used along with the secondorder upwind scheme to solve the governing dimensional equations. The effects of different parameters namely Rayleigh number, the volume fraction of the nanoparticles, and Reynolds number on the streamlines, isotherms and average Nusselt number was investigated. The results showed when Ra increases from $\left(10^{3}\right.$ to $\left.10^{4}\right)$ the average value of the corresponding Nusselt number increases. For increasing the volume fraction along with low Ra values, the average Nusselt number increases, while, for large Ra values and nanoparticles volume fraction of $0.03, \mathrm{Nu}$ has no substantial increase. Selimefendigil and Öztop [46] numerically investigated the MHD-mixed convection within a triangular enclosure provided by a rotating cylinder and filled with $\mathrm{Cu}$-water nanofluid. On the left wall of the cavity, a partial heater was added and the inclined wall on the right was kept at a constant temperature. Other cavity walls and the cylinder are presumed adiabatic. The finite-element method was used to solve the governing equations. The effects on fluid flow and heat transfer of the Grashof number, angular cylinder rotational speed, volume fraction, and Hartmann number were investigated. It was found that the total generation of the entropy, average, and local heat transfer drops with an increase in Hartmann number. The average heat transfer and the total generation of the entropy increase when the cylinder angular velocity and volume fraction increase. Liao and Lin [47] presented a numerical investigation of the natural and mixed convection for a cavity with a stationary and rotating cylinder at the center of the cavity. The solution includes the use of the immersed boundary method. The enclosure walls are cooled and heated bounded by a circular cylinder. The parameters used in the study were Prandtl number (0.07-7.0), Rayleigh number $\left(10^{4}-10^{6}\right)$. The result included the heat transfer quantities for different Rayleigh numbers and Prandtl numbers, and the local and average heat transfer characteristics around the surfaces of both the inner cylinder and outer enclosure. Shih and Chen [48] investigated the effect of high viscous fluid on the fluid flow and thermal fields in an enclosure at different shapes (equilateral triangle, square, and circle) with an inner adiabatic-rotating circular cylinder. The governing equations investigate by finite volume based computational methodology. The enclosure walls were cooled and the inner circular cylinder was heated. The results showed that the triangular cavity showed the highest capacity to dissipate the internal thermal energy over the sidewalls, whereas the poorest outcome was when using the circular cavity. The mixed convection in a square cavity with an adiabatic and rotating-cylinder mounted at the center with two layers of the nanofluid ( $\mathrm{CuO}$-water) and a porous medium was performed numerically by Selimefendigil et al. [49]. The lower and the upper walls are heated and cooled, respectively, while the remaining of the left and right walls are insulated. The governing equations were solved by the finite element method. The numerical investigation included the effects of Rayleigh number $\left(10^{3} \leq \mathrm{Ra} \leq 10^{6}\right)$, nanoparticle size fraction $(0 \leq \phi \leq 0.05)$, angular rotational velocity $(0 \leq \Omega \leq 6000)$, Darcy number ranged from $\left(10^{-2} \leq \mathrm{Da} \leq 10^{-5}\right)$ and different sizes and locations of the cylinder on the fluid flow and heat transfer. The result showed that the rate of heat transfer increased as the value of Rayleigh number, nanoparticle volume fraction, Darcy number and the cylinder angular velocity increased. The influence of the cylinder angular velocity on the enhancement of the heat transfer was more prominent for a larger cylinder. The averaged heat transfer improves linearly with increasing the volume fraction of the nanoparticle for all cases. When the cylinder reaches the upper wall, the local and averaged Nusselt number were enhanced. Selimefendigil and Öztop [50] studied mixed convection in a 3-D enclosure with two inner rotating circular cylinders filled with nanofluids. The finite element method was used for the analysis. The left and right surfaces were heated and cooled respectively, while the other walls and rotating cylinder were kept adiabatic. Water and three types of nanoparticles $\left(\mathrm{TiO}_{2}, \mathrm{Cu}\right.$, and $\left.\mathrm{Al}_{2} \mathrm{O}_{3}\right)$ were examined. The result showed the maximum rate of heat transfer can be obtained at the high value of Rayleigh number with using $\mathrm{Cu}$ water nanofluid. The average Nusselt number was affected by the rotational direction of cylinders. The heat transfer enhancement was $38.10 \%$ for $\mathrm{Cu}$-water compared with water as base fluid. Hussein et al. [51] tested laminar mixed convection in a 2-D laminar trapezoidal enclosure with an inner rotating cylinder and sinusoidal lower wall. The enclosure filled partially with a porous matrix at the bottom which is saturated with nanofluid of (CuO-water) and located at the top of the enclosure. The vertical sidewalls are considered to be adiabatic; however, the enclosure lower and upper walls were kept at hot and cold temperatures, respectively. The dimensionless governing equations were solved using the Galerkin finite element method based on the COMSOL code. The maximum value of stream function for $\mathrm{Ra}=103$ and 105 was shown at $\mathrm{R}=0.2$ and $\mathrm{S}=0.2$. In addition, increasing the inner cylinder radius from 0.1 to 0.3 at $(0.5 \leq \mathrm{S} \leq 0.8)$ and $\left(\mathrm{Ra}=10^{3}\right.$ and $\left.\mathrm{N}=0\right)$ leads to increase the stream function values. The average Nusselt number increased with increasing the numbers of Darcy and Rayleigh, volume fraction, inner cylinder size and the angular rotational velocity rise, while it decreased with increasing the porous layer thickness and the undulations number. Abdulsahib et al. shows the effect of the cylinder rotates is greater on the value of the local Nusselt number when using the low Rayleigh Numbers $[52,53]$.

As seen above, can be concluded that experimental studies less than theoretical studies. Where, nanofluid enclosure investigated experimentally by $\mathrm{Hu}$ et al. $[54,55]$ that heat transfer is experimentally investigated in a square enclosure. In addition, a 2D two-phase Lattice Boltzmann model considering interaction forces between nanoparticles and base fluid is developed for natural convection of nanofluid and is applied to simulate the flow and heat transfer of $\mathrm{Al}_{2} \mathrm{O}_{3}$-water nanofluid in the square enclosure. The numerical results have a good agreement with the experimental results. It is found that the heat transfer of nanofluid is more sensitive to the thermal conductivity than viscosity at low nanoparticle fractions and it is more sensitive to the viscosity than the thermal conductivity at high nanoparticle fractions. While Solomon et al. [56] 
showed that Cavity design is an important aspect in thermal systems, and proper cavity design saves plenty of energy as losses are minimised through better design. In this work, the influence that the Aspect Ratio (AR) of a rectangular cavity filled with nanofluids has on the natural convection process is studied experimentally. Three different cavities with the AR of 1,2 and 4 are fabricated, and the heat transfer performance is studied using two different fluids namely de-ionised water and $\mathrm{Al}_{2} \mathrm{O}_{3} /$ Water nanofluids. It is found that the $\mathrm{AR}$ of the cavity has a significant effect on the heat transfer coefficient and Nusselt number. More importantly, the optimum nanofluid concentration for maximum heat transfer varies with the AR of the cavity. It also found that the Rayleigh number has a strong effect on the Nusselt number as well as nanofluid buoyancy. Abdulsahib and Al-Farhany [57] demonstrated that the increasing temperature difference leads to a noticeable increment in the intensity of the flow.

\section{GOVERNING EQUATION}

The dimensional governing equations of 2-D mixed convection in an enclosure filled with porous medium and nanofluid are given [58, 59].

\subsection{For nanofluid}

$$
\begin{gathered}
\frac{\partial u_{n f}}{\partial x}+\frac{\partial v_{n f}}{\partial y}=0 \\
u_{n f} \frac{\partial u_{n f}}{\partial x}+v_{n f} \frac{\partial u_{n f}}{\partial y}=-\frac{1}{\rho_{n f}} \frac{\partial p}{\partial x}+\frac{\mu_{n f}}{\rho_{n f}}\left(\frac{\partial^{2} u_{n f}}{\partial x^{2}}+\frac{\partial^{2} u_{n f}}{\partial y^{2}}\right) \\
u_{n f} \frac{\partial v_{n f}}{\partial x}+v_{n f} \frac{\partial v_{n f}}{\partial y}=-\frac{1}{\rho_{n f}} \frac{\partial p}{\partial y} \\
+\frac{\mu_{n f}}{\rho_{n f}}\left(\frac{\partial^{2} v_{n f}}{\partial x^{2}}+\frac{\partial^{2} v_{n f}}{\partial y^{2}}\right)+\beta_{n f} g\left(T-T_{c}\right) \\
u_{n f} \frac{\partial T}{\partial x}+v_{n f} \frac{\partial T}{\partial y}=\alpha_{n f}\left(\frac{\partial^{2} T}{\partial x^{2}}+\frac{\partial^{2} T}{\partial y^{2}}\right)
\end{gathered}
$$

\subsection{Porous/nanofluid region}

$$
\begin{gathered}
\frac{\partial u_{p}}{\partial x}+\frac{\partial v_{p}}{\partial y}=0 \\
u_{p} \frac{\partial u_{p}}{\partial x}+v_{p} \frac{\partial u_{p}}{\partial y}=-\frac{1}{\rho_{n f}} \frac{\partial p}{\partial x} \\
+\frac{\mu_{n f}}{\rho_{n f}}\left(\frac{\partial^{2} u_{p}}{\partial x^{2}}+\frac{\partial^{2} u_{p}}{\partial y^{2}}\right)-\frac{\mu_{n f}}{\rho_{n f} k_{p}} u_{p} \\
u_{p} \frac{\partial v_{p}}{\partial x}+v_{p} \frac{\partial v_{p}}{\partial y}=-\frac{1}{\rho_{n f}} \frac{\partial p}{\partial y} \\
+\frac{\mu_{n f}}{\rho_{n f}}\left(\frac{\partial^{2} v_{p}}{\partial x^{2}}+\frac{\partial^{2} v_{p}}{\partial y^{2}}\right)-\frac{\mu_{n f}}{\rho_{n f} k_{p}} v_{p}+\beta_{n f} g(T-T c) \\
u_{p} \frac{\partial T}{\partial x}+v_{p} \frac{\partial T}{\partial y}=\alpha_{e f f}\left(\frac{\partial^{2} T}{\partial x^{2}}+\frac{\partial^{2} T}{\partial y^{2}}\right)
\end{gathered}
$$

\section{CONCLUSIONS}

(1) The dynamic viscosity and effective thermal conductivity of nanofluid have a strong influence on the properties of the convection heat transfer in the cavity. when reducing Prandtl number leads to expand the effects of nanoparticles because effective thermal diffusion will increase. The average Nusselt number increases with volume fraction for all range of Rayleigh numbers.

(2) In a square cavity contains porous media, when increases Darcy number the circulation strength increased, while with increased both Darcy and Grashof numbers the temperature distribution was decreased. Also, with increases in both Rayleigh and Darcy numbers, the average Nusselt number and the values of the stream function increased.

(3) When using two layers under many circumstances no need to fill an enclosure with a porous to achieve the best insulating effect. Also, the heat transfer across the enclosure was higher at the permeable interface. The maximum value of the stream function decreases as the porous layer thickness increases and the Nusselt numbers increase with increase Darcy number.

(4) The average and local Nusselt number values increased with different locations of the inner cylinder with increasing Rayleigh number, due to the big influence of thermal convection. Also, the intensity of the flow circulation and the thickness of the thermal boundary layer around the hot circular cylinder were increased affectedly when increased Rayleigh number. The heat transfer characteristics in the elliptical case are slightly better by comparing the circular and elliptical cylinders.

(5) The convection heat transfer is affected by a rotating cylinder and the cylinder's physical thermal properties are very important, that rotation is more active in the forced convection than in mixed and normal convection. The influence of the angular rotational cylinder on the averaged heat transfer enhancement is more pronounced for large cylinder size. The heat transfer and the average Nusselt number increase as the value of Rayleigh number, Richardson number, Grashof number, nanoparticle volume fraction, Darcy number, and angular rotational speed of the cylinder increases. The triangular cavity had the highest capacity to dissipate internal thermal energy through the sidewalls, whereas the poorest outcome was the circular cavity.

\section{REFERENCES}

[1] Kaviany, M. (2012). Principles of heat transfer in porous media. Springer Science \& Business Media.

[2] Bejan, A. (2013). Convection Heat Transfer. John Wiley \& Sons.

[3] Olayemi, O.A., Khaled, A.F., Temitope, O.J., Victor, O.O., Odetunde, C.B., Adegun, I.K. (2021). Parametric study of natural convection heat transfer from an inclined rectangular cylinder embedded in a square enclosure. Australian Journal of Mechanical Engineering, pp. 1-14. https://doi.org/10.1080/14484846.2021.1913853

[4] Al-Farhany, K., Al-Dawody, M.F., Hamzah, D.A., Hamza, N.H. (2020). Numerical study of nanofluid natural convection in a partially heated tall enclosure. IOP Conference Series: Materials Science and Engineering, 928: 022137. https://doi.org/10.1088/1757$899 x / 928 / 2 / 022137$ 
[5] Abdulkadhim, A., Al-Farhany, K., Abed, A.M., Majdi, H.S. (2020). Comprehensive review of natural convection heat transfer in annulus complex enclosures. Al-Qadisiyah Journal for Engineering Sciences, 13(2): 80-90. https://doi.org/10.30772/qjes.v13i2.633

[6] Vafai, K. (2015). Handbook of Porous Media. CRC Press.

[7] Santra, A.K., Sen, S., Chakraborty, N. (2008). Study of heat transfer augmentation in a differentially heated square cavity using copper-water nanofluid. International Journal of Thermal Sciences, 47(9): 1113 1122. https://doi.org/10.1016/j.ijthermalsci.2007.10.005

[8] Ho, C.J., Chen, M.W., Li, Z.W. (2008). Numerical simulation of natural convection of nanofluid in a square enclosure: effects due to uncertainties of viscosity and thermal conductivity. International Journal of Heat and Mass Transfer, 51(17-18): 4506-4516. https://doi.org/10.1016/j.ijheatmasstransfer.2007.12.019

[9] Abu-Nada, E., Oztop, H.F. (2009). Effects of inclination angle on natural convection in enclosures filled with $\mathrm{Cu}-$ water nanofluid. International Journal of Heat and Fluid Flow, 30(4): $669-678$ https://doi.org/10.1016/j.ijheatfluidflow.2009.02.001

[10] Lin, K.C., Violi, A. (2010). Natural convection heat transfer of nanofluids in a vertical cavity: Effects of nonuniform particle diameter and temperature on thermal conductivity. International Journal of Heat and Fluid Flow, 31(2): 236-245. https://doi.org/10.1016/j.ijheatfluidflow.2009.11.003

[11] Ternik, P., Rudolf, R. (2012). Heat transfer enhancement for natural convection flow of water-based nanofluids in a square enclosure. International Journal of Simulation Modelling, 11(1):

29-39. https://doi.org/10.2507/IJSIMM11(1)3.198

[12] Jahanshahi, M., Hosseinizadeh, S.F., Alipanah, M., Dehghani, A., Vakilinejad, G.R. (2010). Numerical simulation of free convection based on experimental measured conductivity in a square cavity using Water/ $\mathrm{SiO}_{2}$ nanofluid. International Communications in Heat and Mass Transfer, 37(6): 687-694. https://doi.org/10.1016/j.icheatmasstransfer.2010.03.01 0

[13] Oztop, H.F., Mobedi, M., Abu-Nada, E., Pop, I. (2012). A heatline analysis of natural convection in a square inclined enclosure filled with a $\mathrm{CuO}$ nanofluid under non-uniform wall heating condition. International Journal of Heat And Mass Transfer, 55(19-20): 50765086. https://doi.org/10.1016/j.ijheatmasstransfer.2012.05.007

[14] Barik, A.M., Al-Farhany, K. (2019). Numerical investigation of the effect of baffle inclination angle on nanofluid natural convection heat transfer in a square enclosure. Al-Qadisiyah Journal for Engineering Sciences, 12(2): 61-71.

[15] Chopkar, M., Sudarshan, S., Das, P.K., Manna, I. (2008). Effect of particle size on thermal conductivity of nanofluid. Metallurgical and Materials Transactions A, 39(7): 1535-1542. https://doi.org/10.1007/s11661-0079444-7

[16] Sathiyamoorthy, M., Basak, T., Roy, S., Pop, I. (2007). Steady natural convection flow in a square cavity filled with a porous medium for linearly heated side wall (s). International Journal of Heat and Mass Transfer, 50(910):

1892-1901 https://doi.org/10.1016/j.ijheatmasstransfer.2006.10.010
[17] Oztop, H.F. (2007). Natural convection in partially cooled and inclined porous rectangular enclosures. International Journal of Thermal Sciences, 46(2): 149156. https://doi.org/10.1016/j.ijthermalsci.2006.04.009

[18] Revnic, C., Pop, I., Grosan, T., Ingham, D.B. (2009). Natural convection in an inclined square cavity with heated and cooled adjacent walls and filled with a porous medium. The Open Conservation Biology Journal, 1(1): 20-29. https://doi.org/10.2174/1877729500901010020

[19] Al-Farhany, K., Turan, A. (2019). Double-diffusive of natural convection in an inclined porous square domain generalized model. Al-Qadisiyah Journal for Engineering Sciences, 12(3): 151-160.

[20] Abdulkadhim, A., Abed, A.M., Mohsen, A.M., AlFarhany, K. (2018). Effect of partially thermally active wall on natural convection in porous enclosure. Mathematical Modelling of Engineering Problems, 5(4): 395-406. https://doi.org/10.18280/mmep.050417

[21] Al-Farhany, K., Abdulkadhim, A. (2018). Numerical investigation of conjugate natural convection heat transfer in a square porous cavity heated partially from left sidewall. International Journal of Heat and Technology, 36(1): 237-244. https://doi.org/10.18280/ijht.360132

[22] Al-Farhany, K., Abdulkadhim, A. (2018). Numerical simulation for conjugate natural convection in a partially heated rectangular porous cavity. Journal of Engineering and Applied Sciences, 13(16): 6823-6832.

[23] Abdulkadhim, A., Abed, A., Al-Farhany, K. (2018). Computational investigation of conjugate heat transfer in cavity filled with saturated porous media. Frontiers in Heat and Mass Transfer (FHMT). https://doi.org/10.5098/hmt.11.12

[24] Tong, T.W., Subramanian, E. (1986). Natural convection in rectangular enclosures partially filled with a porous medium. International Journal of Heat and Fluid Flow, 7(1): 3-10. $\quad$ https://doi.org/10.1016/0142727X(86)90033-0

[25] Tatsuo, N., Toru, T., Mitsuhiro, S., Yuji, K., Hiroyuki, O. (1986). Numerical analysis of natural convection in a rectangular enclosure horizontally divided into fluid and porous regions. International Journal of Heat and Mass Transfer, 29(6): 889-898. https://doi.org/10.1016/00179310(86)90184-5

[26] Sathe, S.B., Lin, W.Q., Tong, T.W. (1988). Natural convection in enclosures containing an insulation with a permeable fluid-porous interface. International Journal of Heat and Fluid Flow, 9(4): 389-395. https://doi.org/10.1016/0142-727X(88)90005-7

[27] Campos, H., Morales, J.C., Lacoa, U., Campo, A. (1990). Thermal aspects of a vertical annular enclosure divided into a fluid region and a porous region. International Communications in Heat and Mass Transfer, 17(3): 343353. https://doi.org/10.1016/0735-1933(90)90098-5

[28] Bagchi, A., Kulacki, F.A. (2011). Natural convection in fluid-superposed porous layers heated locally from below. International journal of Heat and Mass Transfer, 54(15-16): $3672-3682$ https://doi.org/10.1016/j.ijheatmasstransfer.2011.01.034

[29] Alsabery, A., Saleh, H., Arbin, N., Hashim, I. (2015). Unsteady natural convection in a square cavity partially filled with porous media using a thermal non-equilibrium model. $\quad$ Governing, $\quad 50$ : 12. https://doi.org/10.5281/zenodo.1337625 
[30] Al-Zamily, A.M.J. (2017). Analysis of natural convection and entropy generation in a cavity filled with multi-layers of porous medium and nanofluid with a heat generation. International Journal of Heat and Mass Transfer, 106:

$1218-1231$ https://doi.org/10.1016/j.ijheatmasstransfer.2016.10.102

[31] Kadhim, H.T., Jabbar, F.A., Kadhim, A.A., Jaber, A.K. (2019). Numerical study of nanofluid flow in a square cavity with porous medium using a sinusoidal interface. 2019 4th Scientific International Conference Najaf (SICN), Al-Najef, Iraq, pp. 216-221. https://doi.org/10.1109/SICN47020.2019.9019375

[32] Al-Amir, Q.R., Ahmed, S.Y., Hamzah, H.K., Ali, F.H. (2019). Effects of Prandtl number on natural convection in a cavity filled with silver/water nanofluid-saturated porous medium and non-Newtonian fluid layers separated by sinusoidal vertical interface. Arabian Journal for Science and Engineering, 44(12): 1033910354. https://doi.org/10.1007/s13369-019-04115-y

[33] Yoon, H.S., Ha, M.Y., Kim, B.S., Yu, D.H. (2009). Effect of the position of a circular cylinder in a square enclosure on natural convection at Rayleigh number of 107. Physics of Fluids, 21(4): 047101. https://doi.org/10.1063/1.3112735

[34] Hussain, S.H., Hussein, A.K. (2010). Numerical investigation of natural convection phenomena in a uniformly heated circular cylinder immersed in square enclosure filled with air at different vertical locations. International Communications in Heat and Mass Transfer, 37(8): 1115-1126. https://doi.org/10.1016/j.icheatmasstransfer.2010.05.01 6

[35] Hussein, A.K. (2013). Computational analysis of natural convection in a parallelogrammic cavity with a hot concentric circular cylinder moving at different vertical locations. International Communications in Heat and Mass Transfer, 46: 126-133. https://doi.org/10.1016/j.icheatmasstransfer.2013.05.00 8

[36] Abdulkadhima, A., Al-Farhanyb, K., Abeda, A.M. (2018). Effect of adiabatic circular cylinder on the natural convection heat transfer characterizes in a porous enclosure. Chemical Engineering, 71: 1309-1314. https://doi.org/10.3303/CET1871219

[37] Ravnik, J., Škerget, L. (2015). A numerical study of nanofluid natural convection in a cubic enclosure with a circular and an ellipsoidal cylinder. International Journal of Heat and Mass Transfer, 89: 596-605. https://doi.org/10.1016/j.ijheatmasstransfer.2015.05.089

[38] Hatami, M., Safari, H. (2016). Effect of inside heated cylinder on the natural convection heat transfer of nanofluids in a wavy-wall enclosure. International Journal of Heat and Mass Transfer, 103: 1053-1057. https://doi.org/10.1016/j.ijheatmasstransfer.2016.08.029

[39] Lewis, E. (1979). Steady flow between a rotating circular cylinder and fixed square cylinder. Journal of Fluid Mechanics, 95(3): 497-513. https://doi.org/10.1017/S0022112079001579

[40] Fu, W.S., Cheng, C.S., Shieh, W.J. (1994). Enhancement of natural convection heat transfer of an enclosure by a rotating circular cylinder. International Journal of Heat and Mass Transfer, 37(13): 1885-1897. https://doi.org/10.1016/0017-9310(94)90329-8

[41] Misirlioglu, A. (2006). The effect of rotating cylinder on the heat transfer in a square cavity filled with porous medium. International Journal of Engineering Science, 44(18-19): 1173-1187. https://doi.org/10.1016/j.ijengsci.2006.07.008

[42] Costa, V.A.F., Raimundo, A.M. (2010). Steady mixed convection in a differentially heated square enclosure with an active rotating circular cylinder. International Journal of Heat and Mass Transfer, 53(5-6): 1208-1219. https://doi.org/10.1016/j.ijheatmasstransfer.2009.10.007

[43] Hussain, S.H., Hussein, A.K. (2011). Mixed convection heat transfer in a differentially heated square enclosure with a conductive rotating circular cylinder at different vertical locations. International Communications in Heat and Mass Transfer, 38(2): 263-274. https://doi.org/10.1016/j.icheatmasstransfer.2010.12.00 6

[44] Roslan, R., Saleh, H., Hashim, I. (2012). Effect of rotating cylinder on heat transfer in a square enclosure filled with nanofluids. International Journal of Heat and Mass Transfer, 55(23-24): 7247-7256. https://doi.org/10.1016/j.ijheatmasstransfer.2012.07.051

[45] Matin, M.H., Pop, I. (2014). Numerical study of mixed convection heat transfer of a nanofluid in an eccentric annulus. Numerical Heat Transfer, Part A: Applications, 65(1): 84-105. https://doi.org/10.1080/10407782.2013.812000

[46] Selimefendigil, F., Öztop, H.F. (2014). MHD mixed convection of nanofluid filled partially heated triangular enclosure with a rotating adiabatic cylinder. Journal of the Taiwan Institute of Chemical Engineers, 45(5): 21502162. https://doi.org/10.1016/j.jtice.2014.06.018

[47] Liao, C.C., Lin, C.A. (2014). Mixed convection of a heated rotating cylinder in a square enclosure. International Journal of Heat and Mass Transfer, 72: 922.

https://doi.org/10.1016/j.ijheatmasstransfer.2013.12.081

[48] Shih, Y.C., Cheng, Y.J. (2015). The effect of viscous dissipation on heat transfer in cavities of varying shape due to an inner rotating circular cylinder. Numerical Heat Transfer, Part A: Applications, 68(2): 150-173. https://doi.org/10.1080/10407782.2014.977134

[49] Selimefendigil, F., Ismael, M.A., Chamkha, A. J. (2017). Mixed convection in superposed nanofluid and porous layers in square enclosure with inner rotating cylinder. International Journal of Mechanical Sciences, 124: 95108. https://doi.org/10.1016/j.ijmecsci.2017.03.007

[50] Selimefendigil, F., Öztop, H.F. (2018). Mixed convection of nanofluids in a three dimensional cavity with two adiabatic inner rotating cylinders. International Journal of Heat and Mass Transfer, 117: 331-343. https://doi.org/10.1016/j.ijheatmasstransfer.2017.09.116

[51] Hussein, A.K., Hamzah, H.K., Ali, F.H., Kolsi, L. (2019). Mixed convection in a trapezoidal enclosure filled with two layers of nanofluid and porous media with a rotating circular cylinder and a sinusoidal bottom wall. Journal of Thermal Analysis and Calorimetry. https://doi.org/10.1007/s10973-019-08963-6

[52] Abdulsahib, A.D., Al-Farhany, K. (2020, November). Numerical Investigation of the nanofluid mixed convection on two layers enclosure with rotating cylinder: High Darcy Number Effects. IOP Conference Series: Materials Science and Engineering, 928(2): 022001. https://doi.org/10.1088/1757-899X/928/2/022001

[53] Al-Farhany, K., Abdulsahib, A.D. (2021). Study of 
mixed convection in two layers of saturated porous medium and nanofluid with rotating circular cylinder. Progress in Nuclear Energy, 135: 103723. https://doi.org/10.1016/j.pnucene.2021.103723

[54] Hu, Y., He, Y., Qi, C., Jiang, B., Schlaberg, H.I. (2014). Experimental and numerical study of natural convection in a square enclosure filled with nanofluid. International Journal of Heat and Mass Transfer, 78: 380-392. https://doi.org/10.1016/j.ijheatmasstransfer.2014.07.001

[55] Giwa, S.O., Sharifpur, M., Meyer, J.P. (2020). Experimental study of thermo-convection performance of hybrid nanofluids of $\mathrm{Al}_{2} \mathrm{O}_{3}-\mathrm{MWCNT} /$ water in a differentially heated square cavity. International Journal of Heat and Mass Transfer, 148: 119072. https://doi.org/10.1016/j.ijheatmasstransfer.2019.11907 2

[56] Solomon, A.B., van Rooyen, J., Rencken, M., Sharifpur, M., Meyer, J.P. (2017). Experimental study on the influence of the aspect ratio of square cavity on natural convection heat transfer with $\mathrm{Al}_{2} \mathrm{O}_{3}$ /Water nanofluids.
International Communications in Heat and Mass Transfer, 88: 254-261. https://doi.org/10.1016/j.icheatmasstransfer.2017.09.00 7

[57] Abdulsahib, A.D., Al-Farhany, K. (2020). Experimental investigation of mixed convection on a rotating circular cylinder in a cavity filled with nanofluid and porous media. Al-Qadisiyah Journal for Engineering Sciences, 13(2): 99-108. https://doi.org/10.30772/qjes.v13i2.653

[58] Hussain, S.H., Rahomey, M.S. (2019). Comparison of natural convection around a circular cylinder with different geometries of cylinders inside a square enclosure filled with Ag-nanofluid superposed porousnanofluid layers. Journal of Heat Transfer, 141(2): 022501. https://doi.org/10.1115/1.4039642

[59] Al-Srayyih, B.M., Gao, S., Hussain, S.H. (2019). Effects of linearly heated left wall on natural convection within a superposed cavity filled with composite nanofluidporous layers. Advanced Powder Technology, 30(1): 5572. https://doi.org/10.1016/j.apt.2018.10.007 